\title{
Elaboración de Panes con Agregado de Harina de Arroz Integral y Modelación de sus Atributos Sensoriales a Través de la Metodología de Superficie de Respuesta
}

\author{
Armando Alvis*, Luis Pérez y Guillermo Arrazola \\ Universidad de Córdoba, Programa de Ingeniería de Alimentos, Facultad de Ciencias Agrícolas, \\ Carrera 6 No 76-103, Km 3, vía Cereté, Córdoba-Colombia (e-mail: aalvis2@hotmail.com) \\ ${ }^{*}$ Autor a quien debe dirigirse la correspondencia.
}

Recibido Nov. 22, 2010; Aceptado Dic. 28, 2010; Versión Final recibida Feb. 27, 2011

\begin{abstract}
Resumen
Se estudió la elaboración de pan con agregado de harina de arroz integral a través de 17 formulas experimentales. Esto con el objetivo de encontrar una formulación para la producción de pan que presente mejores propiedades físicas y sensoriales y mejor calidad nutricional. La panificación se llevó a cabo con la hidratación necesaria para mantener la mezcla batida a consistencia constante. Los panes fueron evaluados por sus atributos sensoriales a través de un panel entrenado y el análisis se realizó mediante gráficos de superficies de respuesta para cada una de las variables evaluadas. Se obtuvo correlaciones lineales altamente significativas entre la dureza, elasticidad y volumen específico medidos instrumentalmente y lo determinado por los jueces. Se concluye que los modelos matemáticos desarrollados pueden sustituir la evaluación sensorial de las propiedades físicas evaluadas y determinar la mejor formulación para la producción de pan.
\end{abstract}

Palabras clave: pan, arroz integral, modelado y correlación, atributos sensoriales

\section{Elaboration Bread with Added Rice Flour and Modeling of Sensory Attributes Through Response Surface Methodology}

\begin{abstract}
The production of bread with added rice flour through 17 experimental formulas. This with the aim of finding a formulation for the production of bread that presents better physical and sensory properties and improved nutritional quality. Baking was carried out with the necessary moisture to keep the mix with constant consistency. The sensory attributes of the bread were evaluated by a trained panel and the analysis was performed using response surface graphs for each of the variables evaluated. Highly significant linear correlations were obtained between experimental hardness, elasticity and specific volume and hardness and the characteristics determined by the judges. It is concluded that the mathematical models developed in this work can replace the sensory evaluation of the studied properties and determine the best formulation for the production of bread.
\end{abstract}




\section{INTRODUCCION}

Los productos horneados sobre la base de harina de trigo, son consumidos en forma masiva. Entre ellos, el pan ocupa un lugar preponderante en todo el mundo. Existe, sin embargo, un grupo poblacional que presenta intolerancia a las prolaminas y gluten presentes no sólo en el trigo, sino también en la avena, la cebada y el centeno, conjunto de cereales identificados como TACC. Este serio síndrome, caracterizado por una mala absorción intestinal es llamado enfermedad celíaca y puede llevar a una severa malnutrición (Sánchez et al., 2002). Entre los cereales considerados aptos para ser consumidos por la población que tiene problemas con el gluten (maíz, arroz, sorgo) y que han sido objetos de estudios para intentar sustituir al trigo en la formulación de productos panificados, el arroz es el más utilizado. Esto es debido a que por su bajo contenido en prolaminas (Gujral y Molina, 2004), su carácter hipoalergénico, sabor insípido, bajo contenido en sodio y alto contenido de carbohidratos de fácil digestión, lo transforman en un cereal especialmente apto no sólo para preparar alimentos para celíacos, sino también para intervenir en dietas especiales (Champagne, 1996; Bryant et al., 2001; Kadan y Pepperman, 2002; Sivaramakrishnan et al., 2004; Hagenimana et al., 2006). Veluppillai et al. (2010), estudiaron la posibilidad de sustituir parcialmente harina de trigo con harina de arroz malteado en la fabricación de pan, encontrando una formulación de $65 \%$ de harina de trigo, $35 \%$ de harina de arroz malteado y $2 \%$ de grasa, con un alto grado de aceptación por los consumidores, además la inclusión de esta harina en el pan puede reducir considerablemente el índice glucémico del pan.

El pan es considerado como el más universal de todos los productos horneados y ha sido tan importante en la alimentación humana que se considera como sinónimo de alimento básico en muchas culturas, entre ellas la Europea, Oriental, India y Americana (Lucas, 2008). Este es un producto de gran técnica en su elaboración y puede incorporar una amplia variedad de componentes tales como harina, agua, levadura, sal, azúcar, grasa, leche, huevo, emulsificantes, mejoradores e hidrocoloides, entre otros. Un buen pan debe tener una corteza crujiente, de miga color blanco, de olor apetitoso y con buena conservación; las materias primas que se utilizan tienen una gran influencia en las variaciones de estas características (Clavel, 2001).

Sivaramakrishnan et al. (2004) trabajaron en una serie de ensayos con dos tipos de arroces: grano largo y grano corto. Las harinas de estas muestras fueron utilizadas en la elaboración de pan con aditivos como la hidroxipropilmetilcelulosa. Compararon los resultados obtenidos con harina de trigo (100\%) y con harina de trigo/harina de arroz (50-50), encontrando una importante mejora en las propiedades panificables y en la calidad de textura de miga con las muestras obtenidas a partir del arroz grano largo. Por otra parte Renzetti et al. (2008) trabajando con transglutaminasa mejoraron las propiedades pseudopláticas de los batidos realizados con harina de arroz. También se han realizado estudios con formulaciones para pan sin gluten, reemplazando una parte de harina de arroz por harina de arroz pregelatinizada y se ha observado que la funcionalidad de estas harinas depende del método de cocción utilizado (Sheng, 1995).

En estudios realizado por (Sánchez et al., 2008; Defloor et al., 1991 y Kim y De Ruiter, 1969) sobre la sustitución total de harina de trigo en fórmulas de pan por harinas de harina de arroz extrudidas, ñame y maní desgrasado y por harina de soya respectivamente se reportan resultados muy bajos para el volumen especifico, propiedad física importante en el pan. Esto indica, que la harina de trigo debe sustituirse solo parcialmente en la fórmula para lograr productos de propiedades, atributos y calidad aceptable.

Teniendo en cuenta que los parámetros de textura y sensorial, son definitivos para determinar la calidad global del pan Szczesniak (1975), el objetivo del presente estudio es desarrollar fórmulas de panes con incorporación parcial de harina de arroz integral para modelar sus atributos sensoriales mediante un análisis de superficie de respuesta y a la vez correlacionarlos entre ellos y con los resultados de algunas propiedades físicas y de textura, medidos mediante equipos utilizados en la industria de panificación. 


\section{MATERIALES Y METODOS}

Los panes utilizados para el análisis de propiedades de textura y análisis sensorial fueron panes de molde, que se elaboraron siguiendo el orden aleatorio arrojado por la matriz experimental mostrado en la tabla 1. En las fórmulas, se variaron las proporciones de harina de trigo, harina de arroz integral y grasa y se mantuvieron constantes el resto de los ingredientes (azúcar, sal, agua, levadura y mejorador). En la elaboración de los panes se emplearon harina de trigo comercial marca "3 Castillos", harina de arroz integral marca "Diana"', Margarina (con índice máximo de peróxido $10 \mathrm{meq}-\mathrm{g} \mathrm{O}_{2} / \mathrm{kg}$ grasa e índice máximo de acidez 0,6 $\mathrm{mg} \mathrm{KOH} / \mathrm{kg}$ grasa) marca "La fina", Levadura y mejorador (compuesto por $\alpha$-amilasa, ácido ascórbico y harina de trigo como diluyente) marca "Levapan", sal y azúcar refina y agua potable.

Para la elaboración del pan, la harina de arroz integral se remojó con el $50 \%$ de su peso en agua por 2 horas, para conseguir una mayor absorción de agua y lograr el ablandamiento necesario. Este procedimiento está basado en experiencias anteriores referentes a la producción de pan de corteza suave con harinas de alto contenido de fibra (González, 2004), los ingredientes se mezclaron en una amasadora de espiral NT/70CE marca Tecnopast por 2 min. Posteriormente se adicionó el resto del agua de la fórmula y la grasa continuando el mezclado por $7 \pm 1 \mathrm{~min}$. La masa se colocó en la cámara de fermentación RX-2 marca TNO a una temperatura de $34-35^{\circ} \mathrm{C}$ por un tiempo de 30 minutos, luego la masa se desgasificó en la amasadora de espiral por un tiempo de 3 minutos para lograr un refinamiento y consistencia típica de la masa. La masa refinada se dividió en piezas de $500 \mathrm{~g}$, empleando para ello una balanza mecánica EB 398 marca Berkel, que luego fueron estiradas y enrolladas en forma cilíndrica para colocarlas posteriormente en moldes de panadería previamente engrasados. Los moldes fueron introducidos en la cámara de dilatación a $34-35^{\circ} \mathrm{C}$ (aproximadamente $2 \mathrm{~h}$ hasta que alcanzaron el volumen requerido para ser horneados. Esta operación se realizó en un horno estático B1 marca Thermovet por espacio de 34-36 min. a $180^{\circ} \mathrm{C}$. Luego del horneado los panes se colocaron sobre una rejilla limpia hasta que alcanzaron la temperatura ambiente Los panes se envasaron en bolsas de polietileno por 24 $\mathrm{h}$, hasta realizárseles los análisis instrumentales y sensoriales.

El análisis sensorial se realizó con 7 jueces entrenados en catación de pan (adiestrados durante 6 sesiones en la evaluación de pan con harina de arroz integral), por el método de análisis descriptivo cuantitativo, mediante escala lineal continua de $10 \mathrm{~cm}$ de longitud y de intensidad creciente de un extremo a otro. Los atributos evaluados fueron: desarrollo, poros, olor a cereal integral, dureza, elasticidad, desmoronamiento, sabor a cereal integral y calidad global. Los términos 0 atributos sensoriales empleados fueron tomados de un trabajo realizado con anterioridad sobre la evaluación de pan de corteza suave con harinas de alto contenido de fibra González (2004), generados a partir de una lista previa de descriptores de la literatura y mediante discusión abierta entre catadores y especialistas en el producto.

Los ensayos para evaluar la dureza y la elasticidad se realizaron en un texturometro TA-HDplus $V$ 4.0 de la firma Micro System, mediante el análisis de perfil de textura (TPA), donde se aplicó doble compresión a una rebanada de $25 \mathrm{~mm}$ de espesor hasta un $50 \%$ de su altura a la velocidad de 4 $\mathrm{mm} / \mathrm{s}$ con un tiempo de reposo entre ambas compresiones de $2 \mathrm{~s}$, a partir de los datos fuerza vs tiempo, se calculó la dureza, como la fuerza máxima en la primera compresión y la elasticidad, definida como la distancia que recupera el producto en la segunda compresión y el volumen específico se realizó con un volumenómetro, por desplazamiento de semillas.

Se utilizó un diseño estadístico de experimentos mixto arrojado por el programa estadístico Design -Expert v 6.0.1 (Stat-Ease Inc., 2000). Empleando un D óptimo para la parte correspondiente a las mezclas, variando las proporciones de harina de trigo y arroz integral entre (60 - 90/10 - 40) y un diseño factorial para la parte correspondiente a la proporción de grasa en la fórmula que varió entre $0-6 \%$ (Montgomery, 2002). Los limites inferiores y superiores y el punto central del diseño fueron codificados como $-1,+1$ y 0 respectivamente. La matriz consta de 17 experimentos, cuatro de los cuales son réplicas y se desarrollaron siguiendo el orden aleatorio indicado en la Tabla 1. 
Tabla 1. Corridas experimentales del diseño mixto.

\begin{tabular}{|c|c|c|c|c|c|c|c|}
\hline Corrida & $\begin{array}{c}\mathrm{C}_{1} \\
\text { Harina de } \\
\text { trigo }\end{array}$ & $\begin{array}{c}\mathrm{C}_{2} \\
\text { Harina de } \\
\text { arroz }\end{array}$ & $\begin{array}{c}\mathrm{X}_{1} \\
\text { Grasa }\end{array}$ & Corrida & $\begin{array}{c}\mathrm{C}_{1} \\
\text { Harina de } \\
\text { trigo }\end{array}$ & $\begin{array}{c}\mathrm{C}_{2} \\
\text { Harina de } \\
\text { arroz }\end{array}$ & $\begin{array}{c}\mathrm{X}_{1} \\
\text { Grasa }\end{array}$ \\
\hline 1 & 67.50 & 32.50 & 4.50 & 10 & 82.50 & 17.50 & 1.50 \\
\hline 2 & 60.00 & 40.00 & 6.00 & 11 & 75.00 & 25.00 & 6.00 \\
\hline 3 & 67.50 & 32.50 & 1.50 & 12 & 60.00 & 40.00 & 6.00 \\
\hline 4 & 90.00 & 10.00 & 3.00 & 13 & 60.00 & 40.00 & 0.00 \\
\hline 5 & 75.00 & 25.00 & 3.00 & 14 & 75.00 & 25.00 & 0.00 \\
\hline 6 & 90.00 & 10.00 & 6.00 & 15 & 60.00 & 40.00 & 0.00 \\
\hline 7 & 90.00 & 10.00 & 6.00 & 16 & 90.00 & 10.00 & 0.00 \\
\hline 8 & 90.00 & 10.00 & 0.00 & 17 & 82.50 & 17.50 & 4.50 \\
\hline 9 & 60.00 & 40.00 & 3.00 & \multicolumn{7}{|l}{}
\end{tabular}

La metodología de superficie de respuesta fue aplicada a las variables respuesta usando el software estadístico comercial Design - Expert v 6.0.1 (Stat-Ease Inc., 2000), obteniendo así los modelos de regresión mediante los cuales es posible explicar su comportamiento y la influencia que sobre ellas ejercen los componentes harina de trigo, harina de arroz y la grasa. El gráfico de las superficies de respuesta, el análisis de varianza y los coeficientes de determinación $\left(R^{2}\right)$ fueron generados con el mismo software.

Con la finalidad de establecer si existió correspondencia entre los resultados de las mediciones instrumentales y las evaluaciones sensoriales, se probaron correlaciones entre: la dureza, la elasticidad y el volumen específico medidos por instrumentos y la dureza, la elasticidad y el desarrollo evaluados por jueces. Además se realizó una correlación entre los atributos sensoriales para determinar la interacción entre ellos. Para ello se utilizó el paquete estadístico Verlen, (2009), PASW Statistic 18 (SPSS Inc., 2009).

\section{RESULTADOS Y DISCUSION}

Los resultados del procesamiento estadístico del diseño experimental muestran que el atributo poros, no fue significativo, por lo que no se halló un modelo de regresión que explicara su comportamiento.

Para el resto de los atributos, el análisis de varianza de la regresión resultó significativo para $p<0.0001$. La prueba de falta de ajuste resultó no significativa en todos los casos, los modelos calculados presentaron coeficientes de determinación $R^{2} \geq 0.90$. El análisis de los residuos estandarizados sigue una distribución normal y en todas las ecuaciones, se aprecia que el componente harina de arroz $\left(\mathrm{C}_{2}\right)$ tiene una notable influencia sobre las fórmulas de pan.

Desarrollo. En cuanto al desarrollo del pan, se aprecia de la ecuación 1 que la harina de trigo es quien tiene la mayor influencia en esta variable, debido a que ella posee las proteínas glutelinas y prolaminas, que forman la matriz viscoelástica capaz de retener el gas producido durante el proceso de fermentación, ofreciendo una estructura aireada de la miga y por tanto mayor desarrollo del pan (Marco y Molina, 2008). Además de este componente, la grasa también influyó aunque en menor medida en el desarrollo, ya que la grasa al interactuar con la harina de trigo mejora el efecto lubricante y sella los poros de la red del gluten Lucas (2008); sin embargo, el arroz resulta una carga que no aporta gluten y al aumentar tanto la grasa como el arroz se diluye el gluten presente, afectando el desarrollo del pan, Sin embargo, Gujral y Molina (2004), Marco y Molina (2008), y Pedrosa et al. (2009), empleando mayores proporciones de harina de arroz si encontraron una disminución apreciable de este parámetro físico, por lo que la cantidad utilizada de este componente debe limitarse.

$$
\text { Desarrollo }=7.46 C_{1}+5.39 C_{2}+0.24 C_{1} X_{1}+0.29 C_{2} X_{1} \quad\left[R^{2}=0.95\right]
$$




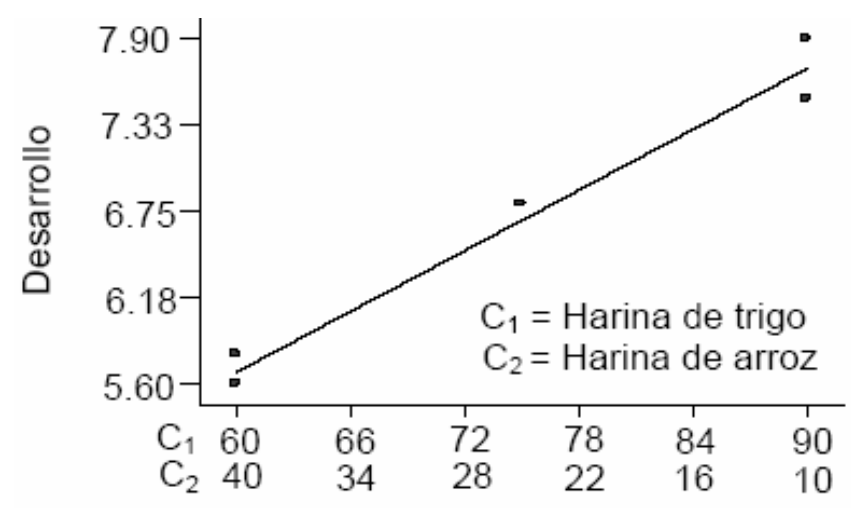

Fig. 1: Gráfico del modelo ajustado para el desarrollo.

Dureza. Se aprecia que ambas harinas aumentan la dureza percibida por los jueces, en mayor medida la harina de arroz, pues esta provoca una miga más compacta, que es un indicador de pan menos aireado y por tanto más duro. Sin embargo, se observa por el término negativo de la ecuación 2, que el efecto de la harina de arroz se atenúa con la adición de grasa en la fórmula.

Dureza $=2.14 C_{1}+5.82 C_{2}-0.86 C_{2} X_{1}+4.75 C_{1} C_{2} X_{1} \quad\left[R^{2}=0.92\right]$

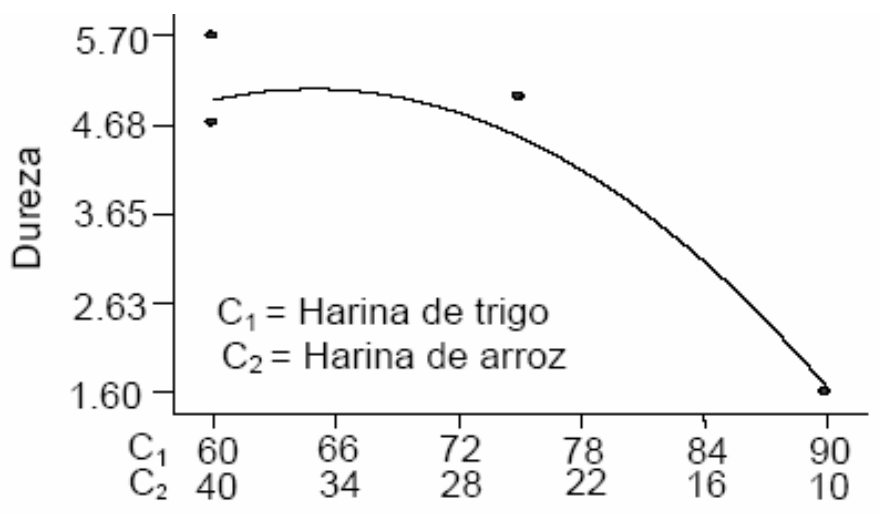

Fig. 2: Gráfico del modelo ajustado para la dureza.

Olor a cereal integral. El olor a cereal integral, se ve influenciado de forma positiva por las harinas, fundamentalmente la de arroz integral, que por su composición tiene un olor más intenso y característico. Al estar unidos estos cereales con la grasa se puede disminuir la intensidad de esta variable, debido a que esta última posee un olor propio que contrarresta el efecto de las harinas, Velluppillai et al. (2010) no encontraron diferencia significativas en pan elaborado con harina de arroz malteado, harina de trigo y grasa.

Olor a Cereal Integral $=3.72 C_{1}+5.82 C_{2}-0.05 C_{1} X_{1}-0.37 C_{2} X_{1} \quad\left[R^{2}=0.94\right]$

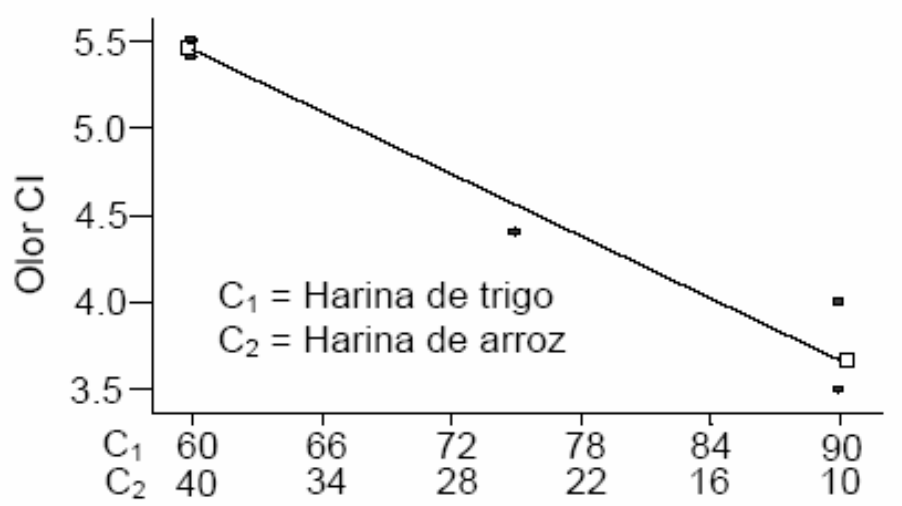

Fig. 3: Gráfico del modelo ajustado para el olor a cereal integral. 
Sabor a cereal integral. El sabor a cereal integral tuvo igual comportamiento que el olor. Éste se vio influenciado por el sabor más intenso de la harina integral que se atenúa también con la adición de la grasa, igual comportamiento encontraron (Velluppillai et al., 2010).

Sabor a cereal integral $=2.81 C_{1}+6.47 C_{2}-0.42 C_{2} X_{1} \quad\left[R^{2}=0.97\right]$

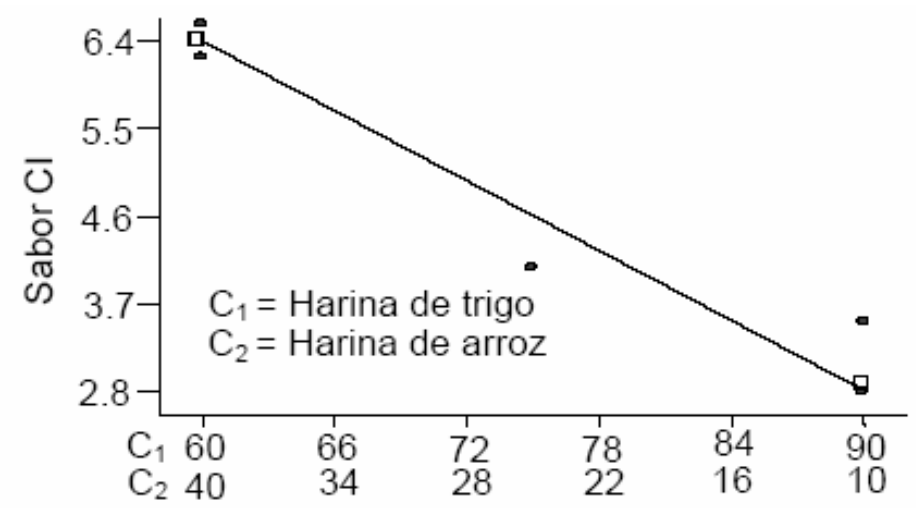

Fig. 4: Gráfico del modelo ajustado para el sabor a cereal integral.

Elasticidad. Se apreció que las harinas son componentes fundamentales en la percepción de este atributo, con mayor influencia la harina de trigo. De acuerdo a la ecuación 5 , la grasa no influyó en los resultados sensoriales, a pesar de que si se apreció en las mediciones instrumentales, por lo que se concluye que estas diferencias no fue percibido por los jueces.

$$
\text { Elasticidad }=7.83 C_{1}+5.63 C_{2}+1.60 C_{1} C_{2} \quad\left[R^{2}=0.90\right]
$$

Una combinación adecuada entre las proporciones de harinas de trigo y arroz integral con la grasa, contribuye a mejorar la elasticidad, tal como se aprecia en la figura 5.

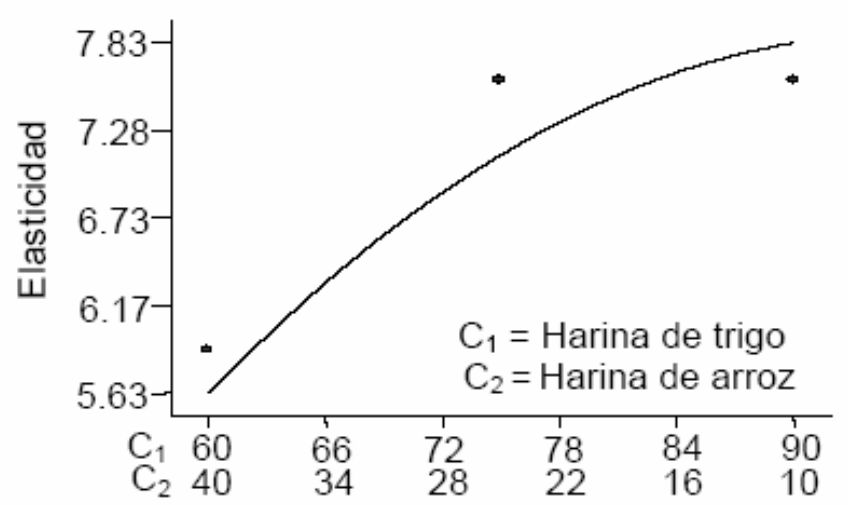

Fig. 5: Gráfico del modelo ajustado para la elasticidad.

Desmoronamiento. Se aprecia en la ecuación 6, un coeficiente bastante elevado de la harina de arroz que afecta de forma positiva esta variable, lo cual no es conveniente. Este incremento del desmoronamiento se debe a que esta harina al no contener gluten hace que se debilite la estructura de la miga. Sin embargo, se aprecia además que una disminución favorable para el valor de esta variable se logra adicionando un porcentaje elevado de harina de trigo y de grasa en la mezcla.

$$
\text { Desmoronamiento }=2.08 C_{1}+6.33 C_{2}-3.35 C_{1} C_{2}-0.74 C_{2} X_{1} \quad\left[R^{2}=0.98\right]
$$




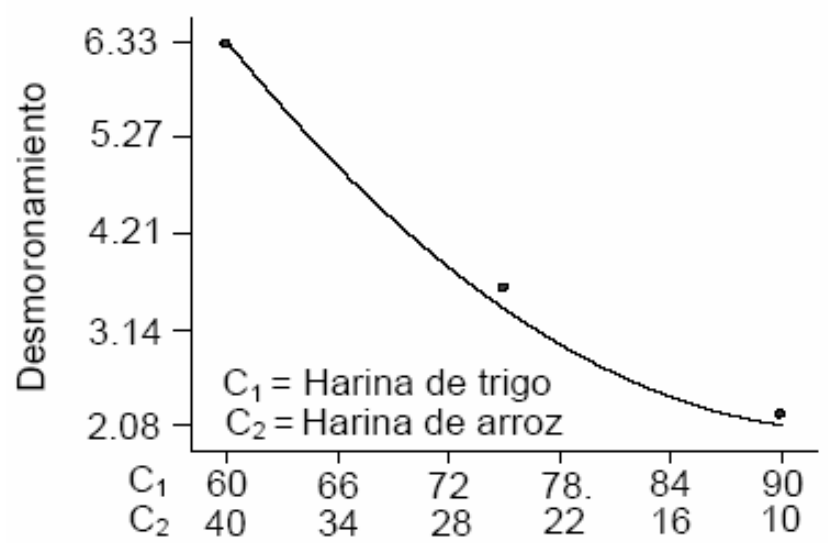

Fig. 6: Gráfico del modelo ajustado para el desmoronamiento.

Calidad global. De acuerdo al modelo que muestra la ecuación 7 , se puede decir que las harinas fueron los componentes fundamentales en la percepción de la calidad por los jueces, con mayor influencia la harina de trigo pero también influyó de forma positiva la grasa. Estos resultados eran de esperar por la influencia que tuvieron estos componentes en todas las variables que resultaron significativas en el modelo y que influyeron definitivamente en la calidad global del pan.

Calidad global $=7.11 C_{1}+5.49 C_{2}+3.03 C_{1} C_{2}+0.22 C_{1} X_{1} \quad\left[R^{2}=0.97\right]$

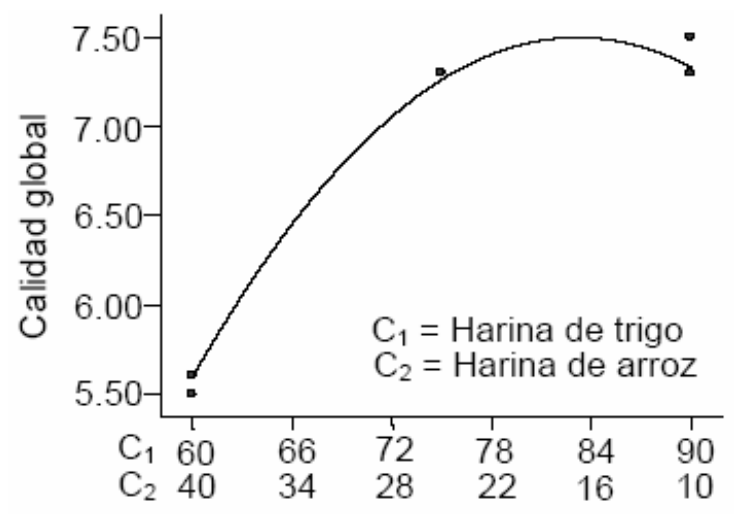

Fig. 7: Gráfico del modelo ajustado para la calidad global.

Las figuras 8,9 y 10, presentan las curvas de ajuste de las correlaciones entre los parámetros instrumentales y los atributos sensoriales.

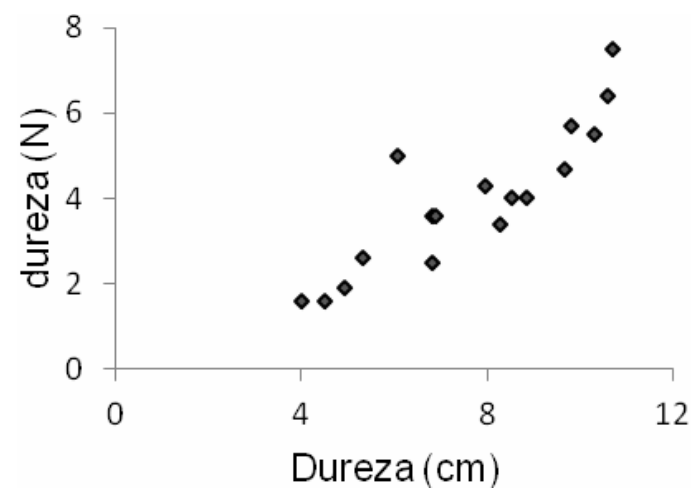

Fig. 8: Diagrama de correlación de la dureza instrumental (d) y sensorial (D) 


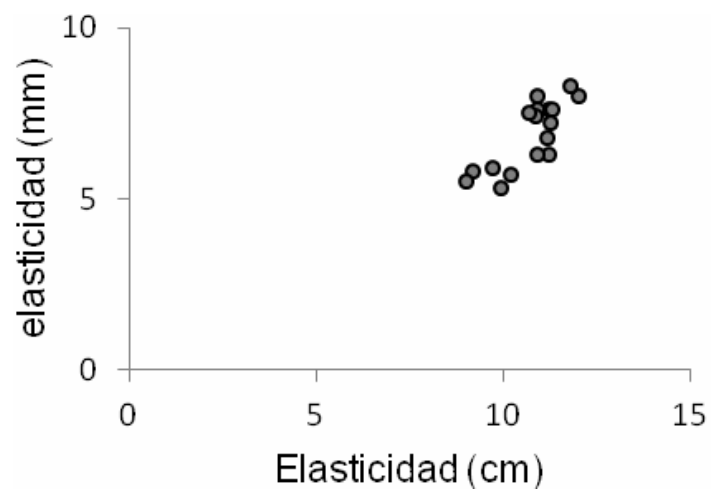

Fig. 9: Diagrama de correlación de elasticidad instrumental (e) y sensorial (E)

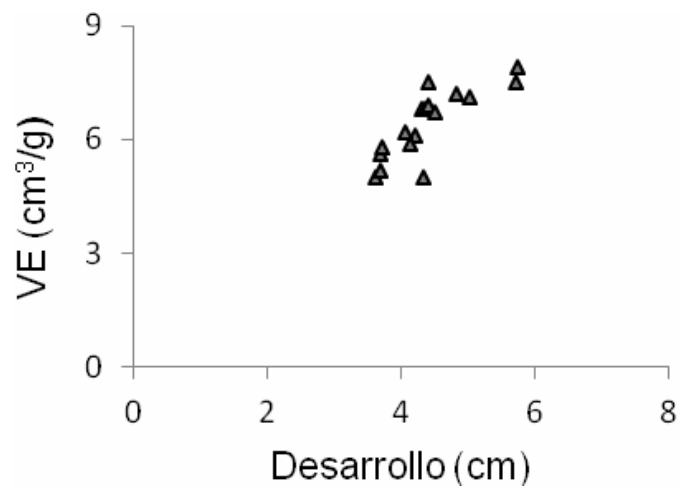

Fig. 10: Diagrama de correlación del volumen específico (VE) y desarrollo (D)

Para cada uno de los parámetros correlacionados se llevó a cabo el mejor ajuste, a través de un modelo lineal con paso obligado por el origen, es decir con intercepto cero. Las ecuaciones de regresión calculadas se muestran en la tabla 2:

Tabla 2. Modelos ajustados a las correlaciones instrumento - sensorial,

\begin{tabular}{|l|l|c|}
\hline Correlación & \multicolumn{1}{|c|}{ Modelos } & Coeficiente de correlación $(r)$ \\
\hline VE $-D$ & VE $=1.459 \mathrm{D}$ & 0.996 \\
\hline $\mathrm{d}-$ Dur & $\mathrm{d}=0.534$ Dureza & 0.981 \\
\hline $\mathrm{e}-\mathrm{E}$ & $\mathrm{e}=0.642 \mathrm{E}$ & 0.995 \\
\hline
\end{tabular}

De acuerdo con estos resultados se puede decir que en el intervalo estudiado, la medición del volumen específico es capaz de predecir los resultados sensoriales del desarrollo del pan, se encontraron valores entre 3.61 y $5.75 \mathrm{~cm}^{3} / \mathrm{g}$ para las diferentes formulaciones, valores muy cercanos al encontrado por Veluppillai et al. (2010) que fue $5.31 \mathrm{~cm}^{3} / \mathrm{g}$ para pan elaborado con harina de arroz malteado y harina de trigo.

A través del cálculo de la dureza y la elasticidad fijadas en este experimento, se encontró valores de dureza en el intervalo de 4.0 a $10.7 \mathrm{~N}$, muy cercano al encontrado por Machado (1996), quien encontró valores de dureza de pan de arroz calculada por TPA entre 14,79 y 104,79 N, señalando que son panes muy duros. También Gujral y Molina (2004) informan resultados alrededor de 14,0 $\mathrm{N}$ para panes elaborados con $100 \%$ harina de arroz, confirmando una firmeza superior a la del pan $100 \%$ de trigo. Por su parte, a través del cálculo de la dureza y la elasticidad mediante el TPA bajo las condiciones fijadas en este experimento, se encontró valores de dureza puede sustituirse la evaluación sensorial de ambos atributos. Todo esto representa aspectos fundamentales de la 
aplicación de las técnicas instrumentales de análisis pues permiten la posibilidad de desarrollar métodos mucho más reproducibles, confiables y rápidos.

En cuanto a las correlaciones entre atributos sensoriales se aprecia que muchos atributos se correlacionaron entre sí, presentando el desarrollo elevadas correlaciones con la dureza de la miga $(r=-0,92)$, la elasticidad $(r=0,92)$, el desmoronamiento $(r=-0,95)$ y la calidad global $(r=$ $0,91)$.

Por su parte, el desmoronamiento obtuvo elevadas correlaciones con la dureza de la miga ( $r=$ $0,93)$ y la elasticidad $(r=-0,92)$.

Finalmente, es preciso resaltar que la calidad del pan, estuvo muy ligada principalmente a los atributos texturales, teniendo un coeficiente de correlación para la elasticidad $(r=0,93)$, el desmoronamiento $(r=-0,93)$ y para la dureza de la miga $(r=-0,83)$. Por tanto, en esta investigación se corroboró y concluyó que los atributos de textura son definitivos para determinar la calidad del pan. Szczesniak (1975), afirma que los parámetros de textura definen la calidad del pan.

\section{CONCLUSION}

Existen correlaciones altamente significativas entre la dureza, elasticidad y volumen específico medidos instrumentalmente y la dureza, elasticidad y desarrollo evaluadas por los jueces, lo que indica un alto poder predictivo del método instrumental para los parámetros de calidad del pan. Además los atributos sensoriales se correlacionan muy bien entre sí, lo que indica que hubo una buena evaluación de los mismos por parte de los jueces.

\section{AGRADECIMIENTOS}

Los autores expresan su total agradecimiento a la Universidad de Córdoba, al grupo de Investigación Procesos y Agroindustria de Vegetales, al Codecyt y al Instituto de Investigaciones para la Industria Alimentaria (IIIA).

\section{REFERENCIAS}

Bryant R. J., R. S. Kadan, E.T. Champagne, B.T. Vinyard y D. Boykin; Functional and digestive characteristics of extruded rice flour, Cereal Chemistry: 78(2), 131-137 (2011).

Champagne, E. T.; Current applications of rice starch, Cereal Foods World: 41(11), 833-838 (1996).

Clavel, R.; El sabor del pan, Molinería y Panadería. (2001), http://www.molineriaypanaderia.com/ tecnica. Accedido: 24 de Abril (2009).

De floor, I. C. De Geest, M. Schllekens, A. Martens y J. Elcour; Emulsifiers and/or extruded starch in the production of breads from cassava, Cereal Chemistry: 68(5), 323-327 (1991).

González, M., Elaboración de un pan de corteza suave con adición de fibra de soya, Tesis de ingeniería químico, Instituto Superior Politécnico "José Antonio Echeverría", La Habana, Cuba (2004).

Gujral, H. y C. Molina; Improvement of the bread making quality of rice flour by glucose. Food Research International. 37(1): 75-81 (2004).

Hagenimana, A., X. Ding y T. Fang; Evaluation of rice flour modified by extrusion cooking. Journal of Cereal Science: 43, 38-46 (2006).

Kadan, R. S. y A. B. Pepperman; Physicochemical properties of starch in extruded rice flours. Cereal Chemistry: 79(4), 476-480 (2002). 
Kim, J. y D. De Ruiter; Bread from non-wheat flours, Food Technology: 22(4), 867-878 (1969).

Lucas, E.; Elaboración de pan. Biotecnología de la fermentación. (2008), http://www.aldeaeducativa.com/panificaciòn/elaboracióndelpan.html, Acceso: 04 de Abril(2009).

Machado, L.; Pão sem glúten: otimização de algumas variáveis de processamento. Tesis de maestría. Departamento de Ingeniería de Alimentos. Universidade Estadual de Campinas, Campinas, Brasil (1996).

Marco, C. y C. Molina; Mejora de la funcionalidad de proteínas de cereales libres de gluten: Aplicación en productos fermentados. Tesis Doctoral. Universidad de Valencia. Valencia, España

Montgomery, L.; Diseño y análisis de experimentos. $2^{\text {da }}$ edición. 225-270. Limusa-Wiley, México, (2002).

Pedrosa M., C. Airoldi y A. El-Dash; Production of acidic extruded rice flour and its influence on the qualities of gluten-free bread. Food Science and Technology 42(3): 618-623 (2009).

Renzetti, S., F. Dal Bello y E. Arendt; Microstructure, fundamental rheology and baking characteristics of batters and breads from different gluten-free flours treated with a microbial transglutaminase, Journal of Cereal Science: 48(1), 33-45 (2008).

Sánchez, H. D., C. A. Osella y M. A. de la Torre; Optimization of gluten-free bread prepared from cornstarch, rice flour, and cassava starch, Journal of Food Science: 67(1), 416-419 (2002).

Sánchez, H. D., R.F. González, C.A. Osella, R.L. Torres y M.A.G. de la Torre; Elaboración de pan sin gluten con harinas de arroz extrudidas, Ciencia y Tecnología Alimentaria: 6(2), 109-116 (2008).

Sheng, D. Y.; Rice-Based ingredients in cereals and snacks, Cereal Foods World: 40(8), 538-540. (1995).

Sivaramakrishnan, H. P., B. Senge y P.K. Chattopadhyay; Rheological properties of rice dough for making rice bread, Journal of Food Engineering: 62: 37-45 (2004).

Stat-Ease Inc; Software for experiment Design - Expert ${ }^{\circledR}$ versión 6,0,1, Wiley, Minneapolis, USA (2000).

Szczesniak, A.; General Foods texture profile revisited - ten years perspective, Journal of Texture Studies: 6(1): 5-17 (1975).

Veluppillai, S., K. Nithyanantharajah, S. Vasantharuba, S. Balakumar y V. Arasaratnam; Optimization of Bread Preparation from Wheat Flour and Malted Rice Flour, Rice Science: 17(1), 51-59 (2010).

Verlen, J.; Predictive Analytics Software PASW Statistic 18, SPSS Inc., Madrid, España, (2009). 